\title{
Pain-Specific Modulation of Hippocampal Activity and Functional Connectivity during Visual Encoding
}

\author{
Katarina Forkmann, ${ }^{1}$ Katja Wiech, ${ }^{3,4}$ Christoph Ritter, ${ }^{1}$ Tobias Sommer, ${ }^{2}$ Michael Rose, ${ }^{2}$ and Ulrike Bingel ${ }^{1,2}$ \\ Departments of ${ }^{1}$ Neurology and ${ }^{2}$ Systems Neuroscience, University Medical Center Hamburg-Eppendorf, D-20246 Hamburg, Germany, ${ }^{3}$ Oxford Centre for \\ Functional Magnetic Resonance Imaging of the Brain (FMRIB), Nuffield Department of Clinical Neurosciences, Nuttfield Division Anaesthetics, University \\ of Oxford, John Radcliffe Hospital, Oxford OX3 9DU, United Kingdom, and ${ }^{4}$ Centre for Pain Research, The University of Bath, Bath BA2 7AY, United \\ Kingdom
}

Acute and chronic pain automatically attract attention and thus interfere with cognitive functioning. Impaired memory is a prominent complaint of patients with chronic pain that substantially contributes to pain-related disability. In this fMRI study, we investigated the specific influence of pain on neural processes of memory encoding in healthy human volunteers using a visual task. To investigate the specificity of the interruptive effect of pain on the encoding of visual objects, objects were presented (1) alone, (2) with painful heat stimuli, or (3) with auditory stimuli that were matched for unpleasantness to the heat stimuli. The interruptive effect of concomitant aversive stimulation on behavioral measures and neural processing was assessed in a categorization task during encoding and in a subsequent recognition task. Pain interfered with object processing and encoding of visual stimuli. On the behavioral level, this resulted in slower reaction times during the categorization task for pain compared with auditory stimuli and in a lower recognition rate in the pain condition but not in the tone condition. Pain catastrophizing amplified this interruptive effect of pain. On the neural level, this painrelated disruption of encoding was associated with reduced activity in the right anterior hippocampus during encoding. Moreover, the hippocampus exhibited reduced functional connectivity with extrastriate regions during painful stimulation relative to auditory stimulation. In summary, our results show a pain-related disruption of visual encoding over and above the unpleasantness of a stimulus, suggesting a pain-specific interruptive mechanism that interferes with an early stage of memory formation.

\section{Introduction}

Because of its biological relevance, pain inherently demands attention and interferes with cognitive processes. This interruptive function of pain (Eccleston and Crombez, 1999) has been demonstrated for acute (Patil et al., 1995; Kuhajda et al., 2002) and chronic (Grisart et al., 2007) pain. Impaired memory is a prominent complaint of patients with chronic pain that substantially contributes to pain-related disability (Schnurr and MacDonald, 1995; McCracken and Iverson, 2001). Subjective complaints about memory performance have been substantiated in behavioral studies showing that working memory (Park et al., 2001; Dick et al., 2008; Luerding et al., 2008; Oosterman et al., 2011) and episodic memory (Grace et al., 1999; Park et al., 2001; Jongsma et al., 2011; Oosterman et al., 2011) can be affected.

Neuroimaging studies have only recently begun to unravel the neurobiological mechanisms underlying the interruptive func-

\footnotetext{
Received June 22, 2012; revised Oct. 19, 2012; accepted Dec. 3, 2012.

Author contributions: K.F., K.W., C.R., T.S., M.R., and U.B. designed research; K.F. performed research; K.F., K.W., C.R., T.S., M.R., and U.B. analyzed data; K.F., K.W., and U.B. wrote the paper.

This work was supported by German Research Foundation Collaborative Research Centre 936/1 and the Federal Ministry of Education and Research Grant 01GQ0808.

The authors declare no competing financial interests.

Correspondence should be addressed to Katarina Forkmann, Department of Neurology, University Medical Center Hamburg-Eppendorf, Martinistrasse 52, D-20246 Hamburg, Germany. E-mail: k.forkmann@uke.uni-hamburg.de. D0I:10.1523/JNEUROSCI.2994-12.2013

Copyright $\odot 2013$ the authors $\quad 0270-6474 / 13 / 332571-11 \$ 15.00 / 0$
}

tion of pain (Bingel et al., 2007; Seminowicz and Davis, 2007a,b; Tiemann et al., 2010; Weissman-Fogel et al., 2011). However, none of these trials specifically addressed memory function. In a previous fMRI study investigating the effect of pain on visual object processing, we found a task-specific modulation of the lateral occipital complex (LOC) (Bingel et al., 2007), a region involved in object processing (Grill-Spector et al., 2001). Intriguingly, a subsequent recognition task revealed compromised memory performance for objects that had been paired with pain, suggesting that pain might affect encoding-specific processes. However, the previous design did not allow to investigate the effects on encoding on a trial-by-trial basis. Furthermore, previous studies on the interruptive function of pain did not control for the unpleasantness of painful stimuli.

Given the inherent unpleasantness of pain, these studies could not exclude whether interference effects of pain on cognitive tasks resulted from the aversive nature of the stimulus in general or its specific nociceptive component.

Here we aimed at investigating whether pain interferes with object encoding and whether this interruptive effect is specific for pain. To this end, we probed the encoding of neutral visual objects and compared the interruptive effect of pain with that of unpleasantness-matched auditory stimuli. Neural and behavioral responses were acquired during an fMRI experiment that was divided into two phases. During encoding, neutral visual objects were presented either alone, in combination with painful heat 
stimuli or unpleasantness-matched auditory stimuli. This phase was followed by a recognition task.

Given the unique biological relevance of pain, we hypothesized that pain would be prioritized over competing stimuli over and above the unpleasantness of the stimulus. We therefore expected reduced performance during encoding and recognition when visual stimuli were paired with painful compared to auditory stimuli. On the neural level, pain was expected to lead to stronger modulations in memory-related brain regions of the medial temporal lobe (MTL), such as hippocampus and parahippocampus (Henson, 2005; Eichenbaum et al., 2007) during encoding. Because the MTL interacts with modality-specific sensory cortices (Cabeza and Nyberg, 2000; Lavenex and Amaral, 2000), we further expected altered neuronal activation in extrastriate regions and functional connectivity between MTL and extrastriate regions during concomitant painful stimulation.

\section{Materials and Methods}

\section{Subjects}

Behavioral and fMRI data were acquired in 28 healthy subjects. Four subjects had to be excluded from the analyses for the following reasons: incidental finding $(n=1)$, depression $>18[n=1$; German version of the Center for Epidemiological Studies Depression Scale, ADS-K (Hautzinger and Bailer, 1993); see below, Psychological questionnaires and executive functioning], microsleep during the task $(n=1)$, or unusual high false-alarm rate $(n=1$, outlier defined as $>2$ SDs). Data from the remaining 24 subjects ( 11 males; all right-handed; mean age, 26.5 years; range, 20-38 years) were included in the final analyses. All subjects had normal pain thresholds at the site of stimulation (Rolke et al., 2006), reported normal hearing, and normal or corrected-to-normal vision. They had no known history of neurological or psychiatric diseases, including recurrent or chronic pain. The study was conducted in accordance with the Declaration of Helsinki and had been approved by the local ethics committee. All participants gave written informed consent to participate and were free to withdraw from the study at any time.

\section{Experimental paradigm}

The study was performed on 2 consecutive days. On the first day, subjects completed preparatory procedures, including the assessment of pain thresholds and pain-related personality traits (see below, Experimental procedures). The actual fMRI experiment was conducted on day 2 and was divided into two parts: (1) the simultaneous presentation of visual and aversive stimuli (encoding phase) and (2) a subsequent recognition task to test for the effect of pain or aversive auditory stimulation on memory encoding. Subjects were informed that the purpose of the study was to investigate the interaction between the perception of neutral visual and aversive stimuli.

\section{Experimental procedures}

Day 1. On day 1, all subjects filled in a number of questionnaires (see below, Psychological questionnaires and executive functioning) assessing general anxiety and depression [ADS-K, State Trait Anxiety Inventory (STAI), trait scale], as well as pain-related psychological processing [Pain Vigilance and Awareness Questionnaire (PVAQ), Pain Anxiety Symptoms Scale (PASS-D), Pain Catastrophizing Scale (PCS)]. Furthermore, participants were tested for two specific subcomponents of executive functioning using the subtests "divided attention" and "flexibility" of the German Test Battery for Attentional Performance (Zimmermann and Fimm, 1992). Next, participants were familiarized with the heat pain
B

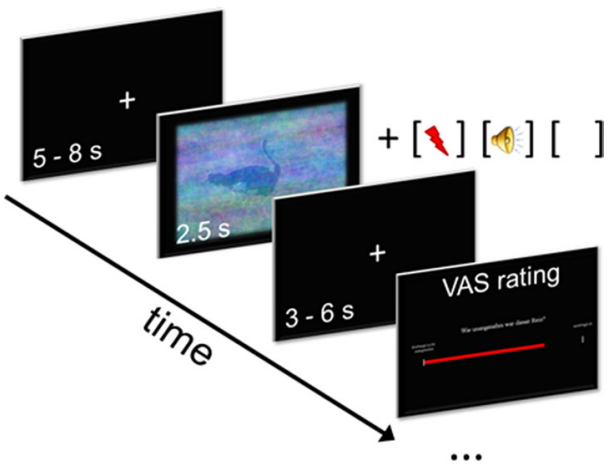

Figure 1. $\quad A$, Experimental protocol. Depicted is the order of preparatory procedures and of the two tasks: the categorization task tional stimulation. Participants rated the unpleasantness of the aversive stimulation after each trial paired with aversive stimula-

and auditory stimuli and underwent a calibration procedure during which they were presented with different stimulation intensities they had to rate for unpleasantness using a visual analog scale (VAS). First, the individual heat pain threshold at the site of stimulus application (left inner forearm, $\sim 12 \mathrm{~cm}$ proximally from the wrist) were determined using the method of limits (Fruhstorfer et al., 1976). Thresholds were obtained using ramped stimuli $\left(1^{\circ} \mathrm{C} / \mathrm{s}\right.$ increase in temperature, starting at a baseline of $35^{\circ} \mathrm{C}$ and with an upper limit of $50^{\circ} \mathrm{C}$ to avoid tissue damage). Participants had to indicate the first painful sensation by pressing a button. Subsequently, all subjects underwent a temperature calibration to determine the individual temperature level corresponding to an unpleasantness level of 70 on the VAS (endpoints: 0, "not unpleasant at all"; 100 , "unbearably unpleasant"). To this end, subjects were presented with stimuli of varying temperature levels around their individual pain threshold (range: $-1^{\circ} \mathrm{C}<$ pain threshold $<+3.5^{\circ} \mathrm{C}$, temperature difference of $0.5^{\circ} \mathrm{C}$, each temperature was applied twice). After each stimulus, participants gave an unpleasantness rating on the VAS that was presented on a computer screen. Subjects indicated the unpleasantness of a stimulus by moving a red bar between the two endpoints of the VAS using two buttons. The temperature that corresponded to an unpleasantness level of VAS 70 was calculated from the ratings provided by a linear regression analysis. Finally, we also introduced a matching procedure to find unpleasantness-matched nociceptive and auditory stimuli (method described below). This procedure was also performed inside the scanner on day 2 .

Day 2. The fMRI experiment was conducted on the second day of the study. First, participants completed the state scale of the STAI. Before the actual experiment, all subjects underwent a number of pretests (i.e., calibration of the individual tone unpleasantness level, matching of tone and pain for unpleasantness; Fig. $1 A$ ) inside the MR scanner while running the same echo-planar imaging (EPI) sequence that was subsequently used during the actual experiment to ensure a comparable testing environment. For the calibration of the individual tone unpleasantness level, auditory stimuli were presented with ascending loudness until a switch from loud to unpleasantly loud noise was indicated by a button press. The tone unpleasantness level was calculated as the mean loudness [decibel sound pressure level (dB SPL)] of the last four of five runs.

To test whether the level of thermal stimulation determined on day 1 still yielded an intensity rating of VAS 70 when presented inside the scanner, five stimuli of the according VAS 70 temperature were presented while running the EPI sequence. Participants again had to indicate the perceived intensity for each stimulus using a VAS with the endpoints labeled as "not painful at all" and "unbearably painful." In the case that the intensity ratings differed significantly from the intensity obtained on day 1 , the temperature was adjusted to correspond to VAS 70 . Subsequently, we performed a matching procedure that had been practiced 
outside the MR scanner on day 1. During this procedure, a heat stimulus corresponding to an individual intensity level of VAS 70 was applied that was immediately followed by an acoustic stimulus. Subjects had to indicate whether the tone stimulus was more, less, or equally unpleasant compared with the heat stimulus. Depending on the subjects' responses, the loudness of the tone in the subsequent trial was automatically reduced or increased. A matching trial terminated if the subject indicated equal unpleasantness for tone and pain stimuli. The individual noise level was calculated as the mean loudness (dB SPL) of five matching trials.

After completion of all pretests, subjects performed the actual encoding task, which was introduced to the subjects as a simple categorization task (duration of $\sim 16 \mathrm{~min}$ ). The task started with 12 practice trials, including four trials of each condition. The practice trials also served to check whether pain and tone stimuli were still matched for unpleasantness. The test trials included 30 images of living objects and 30 nonliving objects, which were reduced in visibility (see below, Visual stimuli).

Visual objects were presented (1) alone ("pic only") or with concurrent (2) painful thermal stimulation ("pain + pic") or (3) auditory stimulation ("tone + pic") that was matched for unpleasantness. All three conditions were presented 20 times in a pseudorandomized order with no more than three consecutive aversive stimuli of the same type. Both pain and auditory stimuli were combined with the same number of living and nonliving objects (i.e., 10 living and 10 nonliving objects). Each trial commenced with the presentation of a white fixation cross (variable duration of $6.5 \pm 1.5 \mathrm{~s}$ ), followed by an image presented for $2.5 \mathrm{~s}$ and another fixation cross (variable duration of $4.5 \pm 1.5 \mathrm{~s}$; Fig. 1B). Participants were instructed to indicate whether the image showed a living or a nonliving object by pressing one of two buttons as quickly as possible without compromising on accuracy (categorization task). Furthermore, in trials in which an aversive stimulus was applied together with the image, subjects rated the unpleasantness of the tone or thermal stimulus using a VAS after a variable duration of 3-6 s in which a fixation cross was presented. Choice, response time (RT), and unpleasantness ratings were recorded as behavioral outcome measures.

To compare the interruptive effect of painful heat and unpleasant auditory stimuli on object encoding, the categorization task was immediately followed by a surprise recognition task (duration of $\sim 14 \mathrm{~min}$ ). Within this task, all images were presented that had been shown in the categorization task and the same number of new images, such as pictures that had not been included in the categorization task or the practice trials. Subjects were instructed to indicate a known (old) or unknown (new) image by pressing one of two buttons (counterbalanced assignment across subjects) as quickly as possible without compromising on accuracy. Overall, 120 images (60 old and 60 new images) were presented for $1.5 \mathrm{~s}$, each with $5 \mathrm{~s}$ between images in which a white fixation cross was displayed. To address the question of object recognition independent of physical attributes such as color, luminance, or texture, all images were shown in full visibility ( $0 \%$ scrambled). Choice and RT were recorded as behavioral outcome measures.

\section{Stimuli}

The presentation of the visual and auditory stimuli, application of the thermal stimuli, and recording of the behavioral data was performed using the software Presentation (www.neurobs.com).

\section{Visual stimuli}

The visual stimuli consisted of pictures showing natural scenes with living or nonliving objects. We selected 60 pictures of living objects (animals such as whales, penguins, cows) and 60 pictures showing nonliving objects (such as cars, buildings, dishes) of neutral valence. In a pilot study, objects had been categorized correctly as living or nonliving within $1500 \mathrm{~ms}$ (data not shown). Sixty images were randomly selected and presented in the categorization task, whereas the recognition task contained all 120 images. To increase the difficulty of the categorization task, the visibility of the images was reduced using a scrambling routine as described previously (Rainer et al., 2001; Rose et al., 2005). In short, the scrambling pattern (i.e., noise) was generated by manipulating a fraction of the image phase before transforming the amplitude and phase components back into image space. Here, $77 \%$ of the phase information was substituted by random phase information, resulting in a "visibility level" of $33 \%$. The outer edges of the images were smoothed with a $28 \mathrm{~mm}$ full-width at half-maximum (FWHM) isotropic kernel to ensure that the images were smoothly embedded into the black background and to reduce transient neural activity evoked by the outer edges. The visual stimuli were presented on a back projection screen located behind the MR scanner. The screen could be seen via a mirror that was attached to the head coil. The pictures had a visual angle of $11.6^{\circ} \times 8.4^{\circ}$ and were displayed for $2.5 \mathrm{~s}$.

\section{Heat pain stimuli}

We used an MR-compatible thermal device (PATHWAY model CHEPS; Medoc) to apply brief painful contact heat stimuli with a total duration of $2.5 \mathrm{~s}$. The heating and cooling rates were set to maximum $\left(70\right.$ and $40^{\circ} \mathrm{C} / \mathrm{s}$, respectively). The CHEPS thermode ( $27 \mathrm{~mm}$ diameter) was attached to the middle of the left inner forearm using a tourniquet. Because we intended to apply heat pain stimuli that corresponded to an individual unpleasantness level of 70 on a $0-100$ VAS but ensure that the overall duration of stimulation was constant across subjects, we had to adjust the stimulus duration at the destination temperature (mean, $2013 \mathrm{~ms}$; range, 1903-2158 ms) depending on the target temperature (mean \pm SD temperature applied, $47.4 \pm 1.7^{\circ} \mathrm{C}$; range, $43.7-50.2^{\circ} \mathrm{C}$ ). The baseline temperature was $35^{\circ} \mathrm{C}$. To ensure the simultaneous perception of visual and heat stimuli and to account for the conduction velocities of pain fibers and a delay between triggering pain stimulation and actual stimulation, the delivery of the heat stimuli was triggered $470 \mathrm{~ms}$ before image presentation as suggested by pilot data (see below, Assessment of stimulus onset latencies).

\section{Auditory stimuli}

The auditory stimulus was created using Audacity 1.3.10-beta (http://www.audacity.sourceforge.net/). The stimulus had a saw-tooth waveform profile with a frequency of $1 \mathrm{kHz}$ and a total duration of $2.5 \mathrm{~s}$ (fading in, $180 \mathrm{~ms}$; fading out, $300 \mathrm{~ms}$ ). The stimulus was monaurally presented through the left headphone (VisuaStim Digital Audio Stimulation; Resonance Technology). Loudness was individually adjusted to correspond to an unpleasantness level of 70 on a $0-100$ VAS (0, "not unpleasant at all"; 100, "unbearably unpleasant"; mean loudness applied, $90.6 \mathrm{~dB}$ SPL; range, $78-106 \mathrm{~dB}$ SPL).

\section{Assessment of stimulus onset latencies}

To ensure the simultaneous perception of aversive stimuli and visual stimuli during the encoding task, we performed a pilot study using a simple detection task. Ten healthy participants were asked to press a button as quickly as possible whenever they (1) notice a blue square presented on a computer screen (detection of a visual stimulus, equals condition pic only in the encoding task), (2) hear a tone (detection of an auditory stimulus, equals condition tone + pic in the encoding task), or (3) perceive a first painful sensation (detection of a nociceptive heat stimulus applied with a thermal device, equals condition pain + pic in the encoding task). All three stimulus types were presented 20 times in a pseudorandomized order with no more than three consecutive stimuli of the same type.

Mean RTs were computed after removing unreasonably short RTs $(<150 \mathrm{~ms})$. A repeated-measures ANOVA with the factor levels (1) visual stimulation (pic only), (2) auditory stimulation ("tone only"), and (3) painful stimulation ("pain only") was used to investigate differences in RTs.

RTs in the detection task differed significantly $\left(F_{(1.238,11.141)}=66.41\right.$, $p<0.001$; corrected according to Greenhouse-Geisser; mean \pm SD, pic only, $456 \pm 93 \mathrm{~ms}$; tone only, $441 \pm 113 \mathrm{~ms}$; pain only, $924 \pm 204 \mathrm{~ms}$ ). Post hoc $t$ tests revealed increased RTs for the painful stimulation compared with both visual stimulation $\left(t_{(9)}=7.95, p<0.001\right)$ and auditory stimulation $\left(t_{(9)}=9.02, p<0.001\right)$. There was no difference between visual and auditory stimulation $\left(t_{(9)}=-1.17, p=0.27\right)$.

Based on our pilot data, we triggered the presentation of painful stimulation during the encoding task $470 \mathrm{~ms}$ before the presentation of the images. This time delay is comparable with peak amplitudes reported in studies using contact heat-evoked potentials (Chen et al., 2001; Le Pera et al., 2002). Using this method, we ensured the simultaneous perception of 
visual and heat pain stimuli and thereby accounted for the conduction velocities of pain fibers as well as the technical delay between triggering pain stimulation and the actual delivering of the stimulation.

\section{Psychological questionnaires and executive functioning}

The interruptive function of pain has been suggested to be moderated by pain-related personality traits, such as pain-related fear (Crombez et al., 1999; Peters et al., 2002) and pain catastrophizing (Grisart and Plaghki, 1999; Van Damme et al., 2004; Vancleef and Peters, 2006a). Furthermore, concepts of top-down control suggest that the capacity of pain to interrupt other ongoing cognitive processes may be associated with executive functioning. To investigate the association of pain-related interference with distinct personality traits that may moderate the interruptive function of pain, participants completed a number of questionnaires assessing these personality traits.

Specifically, participants completed the German version of the following questionnaires on pain-related psychological processing: (1) PVAQ (McCracken, 1997; German version: Lautenbacher et al., 2009); (2) PASS-D (McCracken et al., 1992; German version: Walter et al., 2002); (3) PCS (Bishop et al., 1995; German version: Lautenbacher et al., 2009); (4) Center for Epidemiological Studies-Depression Scale (Radloff, 1977; German version: ADS-K, Hautzinger and Bailer, 1993); and (5) STAI (Spielberger et al., 1983; German version: Laux et al., 1992). Furthermore, we used two subtests (divided attention and flexibility) of the Test Battery for Attentional Performance (TAP), which is a computerized inventory including several subtests to assess different aspects of attention (Zimmermann and Fimm, 1992). All questionnaires and TAP responses were analyzed following the respective manuals.

\section{Analysis of behavioral data}

Behavioral data were automatically recorded and logged by the stimulation program Presentation. All behavioral data analyses were conducted using SPSS version 13.0. Results with a $p<0.05$ are considered as statistically significant. All statistical analyses were performed using two-tailed testing.

Categorization task (encoding phase). The individual categorization performance was defined as the percentage correct classifications separately in each of the three conditions. Trials with RTs longer than $2500 \mathrm{~ms}$ were excluded from additional analyses. The percentage of discarded trials did not differ between conditions $\left(F_{(1.58,49.2)}=2.53, p=0.10\right.$, Greenhouse-Geisser corrected; mean \pm SD, pic only, $5.2 \pm 6.2 \%$; pain + pic, $6.0 \pm 10 \%$; tone + pic, $3.5 \pm 5.8 \%)$. None of the trials had to be excluded as a result of unreasonably short RTs $(<200 \mathrm{~ms})$. Mean RTs were computed for correctly classified images (pooled across living and nonliving objects) separately for each experimental condition after removing extreme outliers (cutoff, $>3$ SDs above mean). Mean unpleasantness ratings were determined for both pain and tone.

Recognition task. Trials with RTs longer than 3 SDs above or below the individual mean RT were excluded from additional analyses. The percentage of discarded trials did not differ between conditions $\left(F_{(2,46)}=\right.$ $1.33, p=0.27$; mean $\pm \mathrm{SD}$, pic only, $2.1 \pm 2.5 \%$; pain + pic, $1.8 \pm 3.2 \%$; tone + pic, $1.0 \pm 2.1 \%$ ). To quantify the recognition performance, we calculated the percentage of correct classifications for each condition (i.e., number of correct classifications in one condition divided by the number of presented pictures in the same condition).

Two separate one-factorial repeated-measures ANOVAs with the factor levels (1) image alone (pic only), (2) image with concurrent painful stimulation (pain + pic), and (3) image with concurrent auditory stimulation (tone + pic) were used to investigate the effects of aversive stimulation on categorization and recognition performances (RTs and accuracy). Significant main effects were followed by post hoc paired $t$ tests. Differences in unpleasantness between pain and tone trials were assessed using a paired $t$ test.

Pain-related psychological processing and executive functioning. Correlations were calculated between the performance measures (i.e., accuracy and RT) and the questionnaire scores as well as divided attention and cognitive flexibility using Pearson's correlation coefficient separately for the categorization and recognition tasks.

\section{Results of behavioral preliminary study}

To pilot our experimental paradigm, we performed a behavioral study in 13 healthy participants (seven males; mean age, 26.2; range, 21-37 years) using the same encoding and recognition paradigm as in the scanning study, except that we did not assess pain-related psychological variables. The unpleasantness ratings for painful and auditory stimulation were comparable for auditory and heat pain stimuli $\left(_{(12)}=0.43, p=0.67\right.$; mean \pm SD, tone + pic, $51.8 \pm 17.4$; pain + pic, $53.7 \pm 17.5)$.

Repeated-measures ANOVAs revealed that the overall categorization accuracy was high and did not differ between the experimental conditions $\left(F_{(2,24)}=0.43, p=0.58\right.$; mean $\pm \mathrm{SD}$, pic only, $97.1 \pm 3.3 \%$; pain + pic, $97.2 \pm 4 \%$; tone + pic, $95.6 \pm 6.7 \%)$. Furthermore, there were no differences in RTs for correct classification during the encoding task $\left(F_{(2,24)}=0.15, p=0.86\right.$; mean $\pm \mathrm{SD}$, pic only, $830 \pm 130 \mathrm{~ms}$; pain + pic, $841 \pm 130 \mathrm{~ms}$; tone + pic, $831 \pm 140 \mathrm{~ms})$.

Critically, a repeated-measures ANOVA (within-subject factor: experimental condition; levels: pic only, pain + pic, tone + pic) revealed significant differences in recognition accuracy between the three conditions $\left(F_{(2,24)}=6.14, p=0.007\right.$; mean $\pm \mathrm{SD}$, pic only, $66.1 \pm 16.7 \%$; pain + pic, $50.7 \pm 10.2 \%$; tone + pic, $63.9 \pm 14.9 \%)$. Recognition rates were significantly lower for images that had been paired with heat pain stimuli compared with images presented alone $\left(t_{(12)}=-4.05, p=0.002\right)$ and images paired with auditory stimuli $\left(t_{(12)}=-2.81, p=0.02\right)$. Differences in recognition rate between tone + pic versus pic only did not reach statistical significance $\left(t_{(12)}=-0.39, p=0.70\right)$. RTs were not significantly different between conditions $\left(F_{(2,24)}=0.97, p=0.40\right.$; mean $\pm \mathrm{SD}$, pic only, $971 \pm 79$ ms; pain + pic, $964 \pm 75 \mathrm{~ms}$; tone + pic, $980 \pm 73 \mathrm{~ms})$.

This study indicated that, although pain and auditory stimuli were equally unpleasant, pain had stronger effects on memory encoding as evident in the impaired recognition rate of visual objects paired with pain.

\section{fMRI data acquisition}

MR scanning was performed on a 3 T MRI system (Siemens Trio) with a standard 16-channel head coil. A total of 42 axial slices (slice thickness, 3 $\mathrm{mm}$ ) per volume were acquired using a gradient EPI T2*-sensitive sequence with the following parameters: repetition time (TR), $2.41 \mathrm{~s}$; echo time (TE), $30 \mathrm{~ms}$; flip angle, $80^{\circ}$; field of view, $210 \times 210 \mathrm{~mm}$. After the functional measurement, an individual high-resolution anatomical image was obtained for each participant using a T1-weighted magnetization-prepared rapid acquisition gradient echo sequence (slice thickness, $1 \mathrm{~mm}$; TR, $2.30 \mathrm{~s}$; TE, $2.98 \mathrm{~ms}$; flip angle, $9^{\circ}$; field of view, $256 \times 256 \mathrm{~mm})$.

\section{Image processing and statistical analyses}

Because of a technical failure, one subject had to be excluded from the fMRI analysis. fMRI results are therefore based on 23 participants. All imaging data were screened for scanner artifacts before data analysis using the toolbox ArtRepair. Image processing and statistical analysis of fMRI data was performed using SPM8 (www.fil.ion.ucl.ac.uk/spm/). After removing the first six volumes to compensate for T1 saturation effects, preprocessing included slice timing and realignment to the first volume. Because pain stimuli often evoke involuntary movements that are correlated with stimulus onset, we corrected for the interaction of head motion and the inhomogeneities of the magnetic field (susceptibility $\times$ movement interaction) using the Unwarping procedure of SPM8. The maximum amount of head motion did not exceed $2.1 \mathrm{~mm}$ in any of the participants.

The anatomical volume was coregistered with the mean echo-planar image and segmented with bias regularization set to medium level. Both structural and functional volumes were normalized to standard Montreal Neurological Institute space using the transformation matrix obtained after the segmentation. Functional images were resampled to a voxel size of $2 \times 2 \times 2 \mathrm{~mm}$ and finally smoothed with an $8 \mathrm{~mm}$ Gaussian kernel with FWHM.

$f M R I$ analyses. Data analysis was performed using the general linear model (GLM). On the subject level, the model for the categorization phase contained three regressors that coded for the three experimental conditions: (1) presentation of images alone (pic only), (2) presentation 
of images with concomitant painful stimulation (pain + pic), and (3) presentation of images with concomitant auditory stimulation (tone + pic). An additional regressor-of-no-interest coded for the rating period after categorization. Each boxcar stimulus function (duration of $2.5 \mathrm{~s}$ ) was convolved with a canonical hemodynamic response function, and data were high-pass filtered with a cutoff period of $128 \mathrm{~s}$. The effects of interest were tested using linear contrasts of the parameter estimates for the three regressors, resulting in a $t$ statistic for each voxel. In a next step, three separate contrast images representing the three conditions pic only, pain + pic, and tone + pic were generated for each participant, which were subsequently included to a second GLM. At the group level, a random-effects approach was used (Friston et al., 1999), treating intersubject variability as a random factor and including nonsphericity.

A second analysis was performed to assess neuronal activation related to successful memory formation [subsequent memory effect (SME)] (Brewer et al., 1998; Kim, 2011). Images were classified in subsequently remembered (hits) and subsequently forgotten (misses) images as assessed in the recognition task. Thus, the subject-specific model contained six regressors of interest: (1) pic only, hits; (2) pic only, misses; (3) pain + pic, hits; (4) pain + pic, misses; (5) tone + pic, hits; and (6) tone + pic, misses, plus one additional regressor-of-no-interest coding for the rating period. The minimum and average number of trials included in the respective regressors were as follows: pic only, hits: mean, 11.3 and minimum, 6; pic only, misses: mean, 8.7 and minimum, 4 ; pain + pic, hits: mean, 9.8 and minimum, 4; pain + pic, misses: mean, 10.2 and minimum, 3; tone + pic, hits: mean, 10.6 and minimum, 3 ; tone + pic, misses: mean, 9.4 and minimum, 4. Note that the pain-specific effect on recognition accuracy led to a lower number of pain + pic, hits trials compared to pic only, hits trials $\left(t_{(23)}=3.23, p=0.004\right)$. No other comparison reached significance.

On the group level, brain regions related to successful encoding regardless of the experimental condition were identified using the six regressors of interest with the respective contrast weights $c=(1,-1,1,-1$, $1,-1)$.

We also contrasted activity associated with successful encoding between the critical experimental conditions (pain + pic vs tone + pic). To test for larger SME in tone trials compared with pain trials, we exclusively masked SME for tone trials [(tone + pic, hits $)>$ (tone + pic, misses $)]$ with the SME for pain trials [(pain + pic, hits $)>$ (pain + pic, misses $)]$ using the contrast weights $c_{\mathrm{SME}}$ tone $=(0,0,1,-1,0,0)$ and $c_{\mathrm{SME} \text { pain }}=(1$, $-1,0,0,0,0)$ for the six regressors of interest.

The threshold for the statistical analyses described above was set to $p<$ 0.05 , familywise error corrected for multiple comparisons. Based on previous studies showing reduced recognition rates and modulations in neuronal activity in medial temporal and ventral visual areas after simultaneous painful stimulation, we performed regions of interest (ROIs) analyses on the following brain regions: fusiform gyrus, LOC, parahippocampal gyrus, and hippocampal regions. We therefore performed an ROI analysis using 20-mm-diameter spheres centered around peak coordinates reported in two previous studies involving similar designs (Powell et al., 2005; Bingel et al., 2007).

Psychophysiological interaction analysis. To further examine the modulatory mechanisms underlying possible differences in disruption by painful or auditory stimulation, a psychophysiological interaction (PPI) analysis (Friston et al., 1997) was performed. A PPI analysis reveals differences in functional connectivity between a particular seed region and all other voxels across the entire brain as a function of a psychological factor.

Here, we conducted a PPI analysis to identify brain regions that showed differential connectivity with the right anterior hippocampus during painful and auditory stimulation that were applied with the images. The right hippocampus was used as the seed region because it showed a pain-specific reduction of neuronal activity [seed region $(x, y$, $z)=(24,-8,-30)$, as identified by the contrast $([$ tone + pic $]>[$ pain + pic]); see Results and Fig. 5A]. The blood oxygenation level-dependent time series was extracted from a sphere located in the right anterior hippocampus (10 $\mathrm{mm}$ diameter, centered on the peak voxel) for every subject individually using the first eigen time series (principal component analysis). The PPI regressor was calculated for each subject as the element-by-element product of the mean-corrected activation of the right hippocampus (extracted time series) and the vector coding for the psychological variable $(-1$ on regressor pain + pic, 1 on regressor tone + pic). Thus, our PPI tested for a pain-specific modulation of the functional connectivity between the right hippocampus and any other brain regions. Finally, the individual contrasts reflecting the interaction between the psychological and physiological variables (PPI regressor) were entered into a one-sample $t$ test.

Because we specifically challenged visual encoding by aversive stimuli, we hypothesized that the impairment of visual object encoding is associated with an impaired connectivity between memory-related regions and visual brain regions such as the LOC and fusiform gyrus, which have been shown previously to be modulated by pain during object processing (Bingel et al., 2007). Therefore, corrections in these regions were based on 20-mm-diameter spheres centered around peak coordinates reported in previous studies (Powell et al., 2005; Bingel et al., 2007).

\section{Results}

\section{Behavioral results}

\section{Unpleasantness rating}

On average, the heat pain threshold was reached at $45.7 \pm 2.6^{\circ} \mathrm{C}$ (mean $\pm \mathrm{SD}$ ), whereas an intensity rating of VAS 70 was yielded with a temperature of $47.4 \pm 1.7^{\circ} \mathrm{C}$. Auditory stimuli that matched the heat pain stimuli in unpleasantness had a loudness level of $90.6 \pm 6.9 \mathrm{~dB}$ SPL. Despite the careful matching of tone and pain stimuli, the tone stimuli were rated as significantly more unpleasant than the heat pain stimuli (VAS tone, $58.0 \pm 14.7$; VAS pain, $\left.49.1 \pm 16.0 ; t_{(23)}=3.77, p<0.001\right)$ during the fMRI experiment.

\section{Categorization task (encoding phase): accuracy and RT}

To examine the differential effect of tone and pain stimuli on categorization accuracy and RTs in the categorization task, repeated-measures ANOVAs (within-subject factor: experimental condition; levels: pic only, pain + pic, tone + pic) were performed for both measures. RTs for images classified correctly differed significantly between the three conditions $\left(F_{(2,46)}=7.56\right.$, $p=0.001$; mean $\pm \mathrm{SD}$, pic only, $1187 \pm 159 \mathrm{~ms}$; pain + pic, $1238 \pm 169 \mathrm{~ms}$; tone + pic, $1141 \pm 144 \mathrm{~ms})$. As revealed by post hoc $t$ tests, the mean RT was longer for images paired with heat pain than for images in the tone + pic condition $\left(t_{(23)}=3.97, p=\right.$ $0.001 ;$ Fig. $2 A)$. The comparison of RTs between pic only and pain + pic trials reached significance $\left(t_{(23)}=-2.06, p=0.05\right)$. Mean RT for pictures paired with tone stimuli were not significantly different from the condition in which the images were presented alone $\left(t_{(23)}=1.79, p=0.09\right)$. Furthermore, categorization accuracy was not significantly different between conditions $\left(F_{(2,46)}=\right.$ $0.95, p=0.39$; mean $\pm \mathrm{SD}$, pic only, $89.4 \pm 8.2 \%$; pain + pic, $87.7 \pm 12.2 \%$; tone + pic, $89.8 \pm 9.7 \%)$.

\section{Recognition task: accuracy and RT}

The average \pm SD false-alarm rate was $8.4 \pm 3.4 \%$ (range, 1.7$15 \%)$. As revealed by a repeated-measures ANOVA (withinsubject factor: experimental condition; levels: pic only, pain + pic, tone + pic), the recognition accuracy differed significantly between the three conditions $\left(F_{(2,46)}=4.52, p=0.02\right.$; mean \pm SD, pic only, $56.7 \pm 13.5 \%$; pain + pic, $48.9 \pm 17.7 \%$; tone + pic, $53.1 \pm 15.1 \%)$. Recognition rates were significantly lower for images that had been paired with heat pain stimuli compared with images presented alone $\left(t_{(23)}=3.02, p=0.006\right.$; Fig. $\left.2 B\right)$. Differences in recognition rate between tone + pic versus pic only and between pain + pic versus tone + pic did not reach statistical significance $t_{(23)}=1.41, p=0.17$ and $t_{(23)}=-1.58$, $p=0.13$, respectively). RTs were not significantly different between conditions $\left(F_{(2,46)}=0.05, p=0.95\right.$; mean $\pm \mathrm{SD}$, pic only, 
$1074 \pm 143$ ms; pain + pic, $1072 \pm 146$ $\mathrm{ms}$; tone + pic, $1077 \pm 160 \mathrm{~ms})$.

Psychological questionnaires and executive functioning

To investigate whether the interference effect of tone and pain varied depending on individual characteristics, we explored the relationship between stimulus-specific interruption of object processing (encoding and recognition) and pain-related psychological processing as well as specific aspects of executive functioning. The pain-related decrease in categorization performance [i.e., (pic only) - (pain + pic)] correlated positively with the median RT of the subtest cognitive flexibility $(r=0.46, p=0.03)$. This indicates that subjects with lower cognitive flexibility (indexed by higher median RTs) had more difficulties in correctly categorizing images presented with pain compared with images presented alone. There was no significant correlation between cognitive flexibility and a tone-related change of performance [i.e., (pic only) - (tone + pic), $r=0.29, p=0.17]$. As expected, the painspecific interference score [i.e., recognition accuracy: (tone + pic) (pain + pic) $]$ correlated positively with the individual pain catastrophizing score ( $r=0.47, p=0.02$; Fig. $2 C)$. The correlation between the PCS and the pain interference score [i.e., recognition accuracy: (pic only) - (pain + pic)] as well as the tone interference score [i.e., recognition accuracy: (pic only) - (tone + pic) $]$ did not reach significance $[($ pic only $)-($ pain + pic $): r=0.31, p=0.14$; (pic only $)-$ (tone + pic): $r=-0.18, p=0.40]$. Furthermore, the pain-specific interference score [i.e., recognition accuracy: (tone + pic) - (pain + pic)] showed a positive correlation with all subscales of the PASS (physiological symptoms of anxiety: $r=0.51, p=0.01$; cognitive symptoms of anxiety: $r=0.41, p=0.04$; escape/avoidance: $r=0.40$, $p=0.05)$. Together, higher levels of pain anxiety and pain catastrophizing seem to increase the interference effect of pain. All other correlations did not reach statistical significance.

\section{Imaging results}

Pain-related activity

Painful contact heat stimulation $[($ pain + pic $)>$ (pic only)] resulted in activations in brain regions implicated in pain perception (Apkarian et al., 2005). This included the contralateral secondary somatosensory cortex (SII), inferior parietal and cingulate cortices (including anterior and midcingulate cortices), precuneus, insula, and cerebellum. Subcortical activations were found in the right caudate and bilateral thalamus (Table 1, top).

Painful stimulation compared with unpleasant auditory stimulation $[($ pain + pic $)>($ tone + pic $)]$ specifically activated contralateral SII, bilateral insula, and cingulate areas (anterior and midcingulate). Again, subcortical activations included the thalamus and the right putamen (Table 2, top).

\section{Tone-related activity}

The unpleasant auditory stimulation $[($ tone + pic $)>($ pic only $)]$ led to strong activations across the bilateral superior temporal gyrus, including primary (Heschl's gyrus) and secondary auditory areas. Additional activations were found in inferior parietal and cingulate cortices, the cerebellum bilaterally, the precuneus, frontal areas (middle and superior), and the left thalamus (Table 1, bottom).

Auditory stimuli compared with nociceptive stimuli $[($ tone + pic $)>($ pain + pic $)]$ specifically activated the bilateral superior tem-
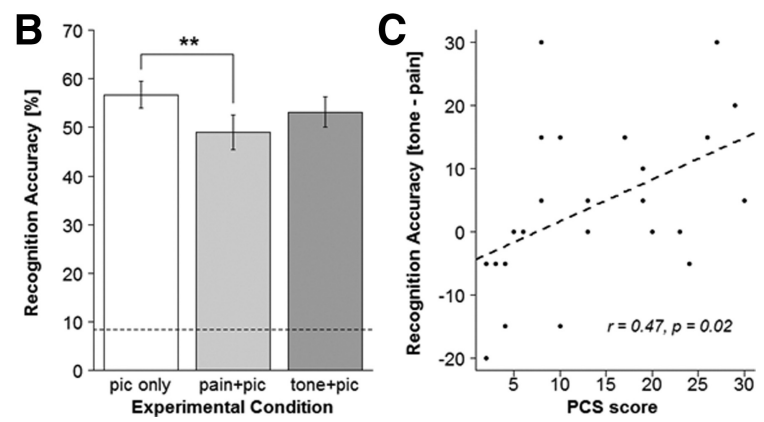

Figure 2. Behavioral effects of simultaneously presented aversive stimulation. $\boldsymbol{A}$, Mean RTs for correctly categorized images were increased for images presented with painful stimulation compared with unpleasant auditory or no stimulation. $\boldsymbol{B}$, Concurrent painful stimulation reduced the accuracy in a subsequent recognition task, whereas unpleasant auditory stimulation did not affect formance. The dashed line indicates the mean false-alarm rate. C, The pain-specific interruptive effect (defined as the difference in recognition accuracy between the tone + pic and pain + pic conditions) was augmented in participants scoring

poral gyrus, including Heschl's gyrus, the temporal pole, inferior frontal and angular gyrus, as well as the brainstem (Table 2, bottom).

\section{Encoding-related activity}

At first, we probed brain regions related to successful memory encoding regardless of the experimental condition. A subsequent memory effect, that is, increased activations for subsequently remembered compared with subsequently forgotten images (hits $>$ misses $)$, was present in the bilateral anterior hippocampus $[(x, y$, $z)$ : left hippocampus, $(-30,-18,-16), t=5.31, p<0.05$ corrected; right hippocampus, $(34,-12,-18), t=5.18, p<0.05$ corrected $]$ and the left anterior parahippocampus $[(-24,-18$, $-18), t=5.11, p<0.05$ corrected; see Fig. $4 A]$.

\section{Effect of pain and unpleasant tone on visual processing} and encoding

In a next step, we tested whether the concurrent application of painful heat stimuli or unpleasantness-matched auditory stimuli modulated neuronal activation in regions related to encoding and visual object processing.

Concurrent painful stimulation $[$ pain + pic $)<$ (pic only $)]$ substantially reduced neuronal activity in the medial frontal gyrus $[(x, y, z):(4,30,-18), t=6.33, p<0.05$ corrected $]$, the superior medial frontal gyrus $[(14,48,4), t=5.32, p<0.05$ corrected], the left anterior and posterior parahippocampal gyrus $[(-22,-4,-26), t=4.3 ;(-30,-38,-14), t=4.9, p<$ 0.05 corrected $]$, as well as the right fusiform gyrus $[(32,-40$, $-18), t=3.6, p<0.05$ corrected; Fig. 3A]. Simultaneous auditory stimulation $[($ tone + pic $)<$ (pic only) $]$ also significantly reduced neuronal activity in the right posterior hippocampus $[(32,-30,-10), t=3.5, p<0.05$ corrected $]$ and the left posterior parahippocampal gyrus $[(-30,-36,-14)$, $t=3.5, p<0.05$ corrected].

As hypothesized, we found a pain-specific reduction within the MTL as revealed by the contrast $[$ (pain + pic $)<($ tone + pic)]. Specifically, we observed a stronger reduction for concurrent painful stimulation compared with unpleasant auditory stimulation in the right anterior hippocampus $[(24,-8,-30)$, $t=3.35, p<0.05$ corrected; Fig. $4 A$ ].

The same activation pattern was observed in the medial frontal gyrus that showed reduced activation $[(18,46,4), t=6.32, p<0.05$ corrected]. The reverse contrast $[($ tone + pic $)<($ pain + pic $)]$ revealed no significant reduction of activity in the MTL.

In addition, the right anterior hippocampus showed a stronger subsequent memory effect under concurrent auditory [(tone 


\begin{tabular}{|c|c|c|c|c|c|c|c|}
\hline \multirow[b]{3}{*}{ Region } & \multicolumn{6}{|c|}{ Coordinates (in mm) } & \multirow[b]{3}{*}{ Voxel level ( $T$ ) (left/right) } \\
\hline & \multicolumn{3}{|l|}{ Left } & \multicolumn{3}{|c|}{ Right } & \\
\hline & $x$ & $y$ & $z$ & $x$ & $y$ & $z$ & \\
\hline \multicolumn{8}{|l|}{ Pain-related neuronal activation $[$ (pain + pic) $>$ (pic only) $]$} \\
\hline Insula, posterior & -34 & -18 & 12 & 38 & -16 & 18 & $9.2 / 10.2$ \\
\hline Insula, anterior & -30 & 16 & 6 & & & & $10.0 /-$ \\
\hline MCC/SMA & -4 & 16 & 44 & & & & $9.7 /-$ \\
\hline SII & & & & 62 & -18 & 22 & $-/ 8.8$ \\
\hline LOC, superior division & -18 & -60 & 52 & & & & $8.6 /-$ \\
\hline Middle frontal gyrus & & & & 30 & 0 & 54 & $-/ 7.1$ \\
\hline Cerebellum & & & & 32 & -56 & -32 & $-/ 7.1$ \\
\hline Inferior parietal cortex & -44 & -36 & 44 & 38 & -40 & 42 & $6.6 / 5.7$ \\
\hline Thalamus & -14 & -20 & 8 & 18 & -14 & 8 & $6.0 / 5.7$ \\
\hline DLPFC & -38 & 32 & 28 & & & & $6.3 /-$ \\
\hline Caudate nucleus & & & & 16 & 16 & 0 & $-/ 5.5$ \\
\hline \multicolumn{8}{|l|}{ Tone-related neuronal activation $[$ (tone + pic) $>$ (pic only) $]$} \\
\hline Superior temporal gyrus (including Heschl's gyrus) & -44 & -28 & 6 & 56 & -20 & 8 & $16.8 / 19.4$ \\
\hline Cerebellum & -40 & -50 & -32 & 34 & -56 & -32 & $7.5 / 8.8$ \\
\hline LOC, superior division & -20 & -58 & 52 & & & & $8.5 /-$ \\
\hline Premotor cortex/SMA & -4 & 10 & 54 & & & & $8.1 /-$ \\
\hline Supramarginal gyrus, posterior division & -46 & -44 & 54 & 44 & -44 & 50 & $8.0 / 5.3$ \\
\hline Thalamus & -4 & -18 & 12 & & & & $7.8 /-$ \\
\hline Posterior cingulate & -2 & -40 & 22 & & & & $7.4 /-$ \\
\hline Middle frontal gyrus & & & & 28 & -2 & 54 & $-/ 7.0$ \\
\hline DLPFC & -40 & 30 & 30 & & & & $6.7 /-$ \\
\hline Cingulum & -4 & -18 & 34 & & & & $5.7 /-$ \\
\hline Precuneus & & & & 12 & -62 & 36 & $-/ 5.6$ \\
\hline Caudate nucleus & & & & 20 & 20 & 4 & $-/ 5.3$ \\
\hline
\end{tabular}

MCC, Midcingulate cortex; SMA, supplementary motor area; DLPFC, dorsolateral prefrontal cortex.

Table 2. Stimulus-specific neuronal activations ( $p<0.05$, corrected)

\begin{tabular}{|c|c|c|c|c|c|c|c|}
\hline \multirow[b]{3}{*}{ Region } & \multicolumn{6}{|c|}{ Coordinates (in mm) } & \multirow[b]{3}{*}{ Voxel level ( $T$ ) (left/right) } \\
\hline & \multicolumn{3}{|l|}{ Left } & \multicolumn{3}{|c|}{ Right } & \\
\hline & $x$ & $y$ & $z$ & $x$ & $y$ & $Z$ & \\
\hline \multicolumn{8}{|c|}{ Pain-specific neuronal activation $[$ (pain + pic) $>$ (tone + pic $)]$} \\
\hline SII & & & & 40 & -18 & 22 & $-/ 10.3$ \\
\hline Insula, anterior & -34 & 4 & 12 & 36 & 8 & 10 & $10.3 / 10.1$ \\
\hline Insula, posterior & -34 & -18 & 14 & & & & $8.1 /-$ \\
\hline Precentral gyrus & & & & 58 & 6 & 8 & $-/ 7.3$ \\
\hline Insula & & & & 38 & 2 & -8 & $-/ 6.4$ \\
\hline \multicolumn{8}{|c|}{ Tone-specific neuronal activation $[$ (tone + pic $)>($ pain + pic $)]$} \\
\hline Superior temporal gyrus (including Heschl's gyrus) & -44 & -28 & 6 & 64 & -26 & 10 & $16.3 / 18.4$ \\
\hline Inferior frontal gyrus & -54 & 30 & -6 & & & & $6.5 /-$ \\
\hline Precentral gyrus & -2 & -34 & 70 & & & & $6.0 /-$ \\
\hline Brainstem & & & & 14 & -30 & -32 & -15.8 \\
\hline Temporal pole & & & & 48 & 18 & -32 & $-/ 5.6$ \\
\hline Angular gyrus & -52 & -56 & 20 & & & & $5.4 /-$ \\
\hline
\end{tabular}

+ pic, hits $)>($ tone + pic, misses $)]$ compared with concurrent painful stimulation $[$ (pain + pic, hits $)>$ (pain + pic, misses $)$; $(32,-8,-26), t=3.71, p<0.05$ corrected].

No significant effect in visual ROIs was observed for any of these contrasts.

\section{PPI analysis}

To explore the modulatory mechanisms underlying the differential effects of pain and auditory stimulation on encoding, we investigated condition-specific changes in functional connectivity of the hippocampus using PPI analysis. The PPI analysis tested for a painspecific modulation of the functional connectivity between the right hippocampus $[(24,-8,-30)$; see Fig. $5 A]$ that showed a painspecific decrease in activation (see above) and any other brain re- gion. This analysis revealed reduced functional connectivity between the right hippocampus and ventral visual areas, for example, fusiform gyrus $[(36,-62,-16), t=4.59, p<0.05$ corrected $]$ and lingual gyrus $[(-34,-80,-11), t=3.73, p<0.05$ corrected $]$, both regions that correspond to the LOC, as well as the calcarine gyrus [BA 17, primary visual cortex; $(6,-96,-2), t=3.76, p<0.05$ corrected] during pain + pic trials compared with tone + pic trials (Fig. 5B). In contrast, no reduction in functional connectivity was observed during auditory compared with painful stimulation.

\section{Discussion}

This study explored the neuronal mechanisms underlying the disruptive capacity of pain on memory in a visual encoding task. 
To test for pain-specific effects, the influence of pain on the encoding of visual objects was compared with that of an auditory stimulus of matched unpleasantness. Our study revealed four novel findings. (1) Painful stimulation slowed down RTs during encoding and compromised the performance in a surprise recognition task. (2) These behavioral findings were associated with changes on the neural level: pain interfered with visual encoding, indexed by a pain-specific reduction in activation of the right anterior hippocampus as a region related to successful visual encoding. (3) Furthermore, the hippocampus showed reduced functional connectivity with extrastriate regions during painful stimulation relative to unpleasantness-matched auditory stimulation. (4) Finally, the interruptive effect of pain varied depending on painrelated psychological traits because individuals scoring high on pain catastrophizing and pain-related anxiety showed more painspecific impairment of recognition performance.

\section{Specificity of the interruptive effect of pain on visual encoding}

Our findings of compromised performance during concurrent painful stimulation confirm previous studies using experimentally induced (Eccleston and Crombez, 1999; Vancleef and Peters, 2006a,b; Bingel et al., 2007; Tiemann et al., 2010) and chronic (Grace et al., 1999; Park et al., 2001; Dick et al., 2008) pain. However, these studies could not rule out that the interference effect of pain on cognitive tasks resulted from the aversive nature of the stimulus in general or the specific nociceptive component of pain. Behavioral studies indicate that the ability of a stimulus to disrupt ongoing processes is critically determined by its threat value (Eccleston and Crombez, 1999). We therefore intended to match the threat value (operationalized here as unpleasantness) of aversive auditory and painful stimuli to investigate whether the nociceptive component specifically increases the interruptive function and to identify a pain-specific neuronal mechanism that allows pain to be prioritized over other competing stimuli.

Unpleasant noise has been shown previously to reduce hippocampal activity and to attenuate the recognition performance in a picture encoding task (Hirano et al., 2006). However, our study demonstrates that noxious stimuli were more disruptive during the encoding of visual stimuli than unpleasantnessmatched auditory stimuli. Importantly, the specificity of painrelated disruption was found on the behavioral (Fig. 2) and the neuronal (Figs. 3, 4) levels. It should be noted that, despite a careful matching procedure, auditory stimuli were rated as more unpleasant than painful stimuli. However, this slight imbalance strengthens rather than weakens our findings because painful stimuli were even more disruptive, although they were slightly less unpleasant. This finding supports the notion that the nociceptive component of pain is an additional significant feature that increases its interruptive function and enables pain to be prioritized among competing processes (over and above unpleasantness per se) because of its inherent link to potential or actual tissue damage.

Recent studies have shown improved memory performance for neutral items that had been encoded in an emotionally arousing context (e.g., aversive or painful stimuli) compared with those encoded in a neutral context (Dunsmoor et al., 2012; Schwarze et al., 2012). However, in these studies, an increase in arousal was induced shortly after stimulus onset, and retrieval of the presented stimuli was tested after a retention interval of $24 \mathrm{~h}$. In contrast, in our study, the visual and the (potentially) disturbing nociceptive stimuli were presented simultaneously. Our findings are therefore in line with the notion that acute stress impairs memory during immediate recall (Kirschbaum et al., 1996; Jelici et al., 2004).

\section{Modulatory influence of catastrophizing}

Moreover, we show that high catastrophizers were more distracted by pain and exhibited lower recognition performance. This augmentation of the interruptive function of pain on memory encoding by pain catastrophizing is in accordance with results from previous studies (Van Damme et al., 2004; Tiemann et al., 2010). Although our task was not designed to explore the subprocesses involved, this finding may reflect particular problems in disengaging from pain in high catastrophizers as suggested by van Damme et al. (2004). It should be noted that the pain catastrophizing scores obtained in the healthy volunteers investigated here were considerably lower than scores commonly found in chronic pain patients (Dick and Rashiq, 2007). It can therefore be assumed that the effects observed in our study underestimate rather than overestimate the potentially detrimental consequences of pain catastrophizing on memory function in chronic pain patients.

\section{Pain-specific modulation of hippocampal activity during visual encoding}

Using a visual encoding paradigm, we found that simultaneously presented unpleasant stimulation reduced neuronal activations in medial temporal regions during the encoding of neutral images. More specifically, the left parahippocampal gyrus and the right fusiform gyrus showed less activation under concurrent painful stimulation, whereas unpleasant auditory stimulation reduced activity in the right posterior hippocampus and the left parahippocampal gyrus, albeit to a lesser extent.

Importantly, when directly comparing the modulatory effects of painful and auditory stimulation, we observed a pain-specific reduction of activity in the right anterior hippocampus. MTL regions and in particular the hippocampus are pivotal for the encoding of episodic memory (Henson, 2005; Eichenbaum et al., 2007). Intriguingly, the site of pain-specific modulation corresponds nicely to the site showing a subsequent memory effect in our visual encoding task (Fig. $4 A$ ). Because the right anterior hippocampus was related to successful encoding, the painspecific reduction of activation observed in the right hippocampus most likely reflects reduced encoding-related activity during painful compared with auditory stimulation. 
A

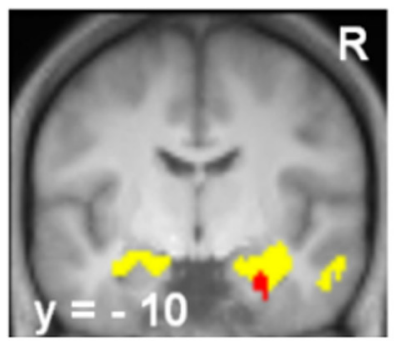

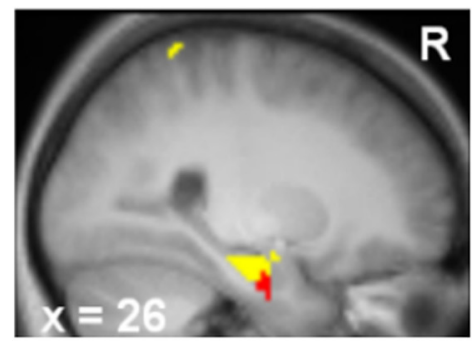
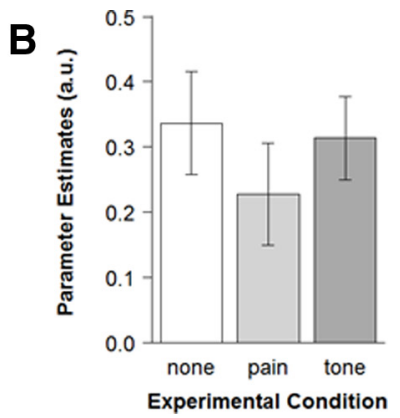

Figure 4. Pain-specific modulation in regions related to successful memory encoding. $\boldsymbol{A}$, Subsequently remembered images (averaged across conditions) significantly activated the right hippocampus (shown in yellow) during encoding as revealed by the contrast (subsequent hits $>$ subsequent misses; for visualization purposes, thresholded at $p<0.001$ uncorrected). Intriguingly, we noted a pain-specific reduction of neuronal activity in the right anterior hippocampus during simultaneous painful stimulation compared with unpleasant auditory stimulation [(pain + pic) $<$ (tone + pic)] that overlapped with the site showing a subsequent memory effect (colored in red). Clusters are overlaid on the group mean T1-weighted image; for visualization purposes, thresholded at $p<0.01$ uncorrected. $B$, Parameter estimates for a $5 \mathrm{~mm}$ sphere located in the right anterior hippocampus $[(x, y, z)=(24,-8,-30)]$; plotted for illustration purpose. Error bars indicate the SEM. R, Right.
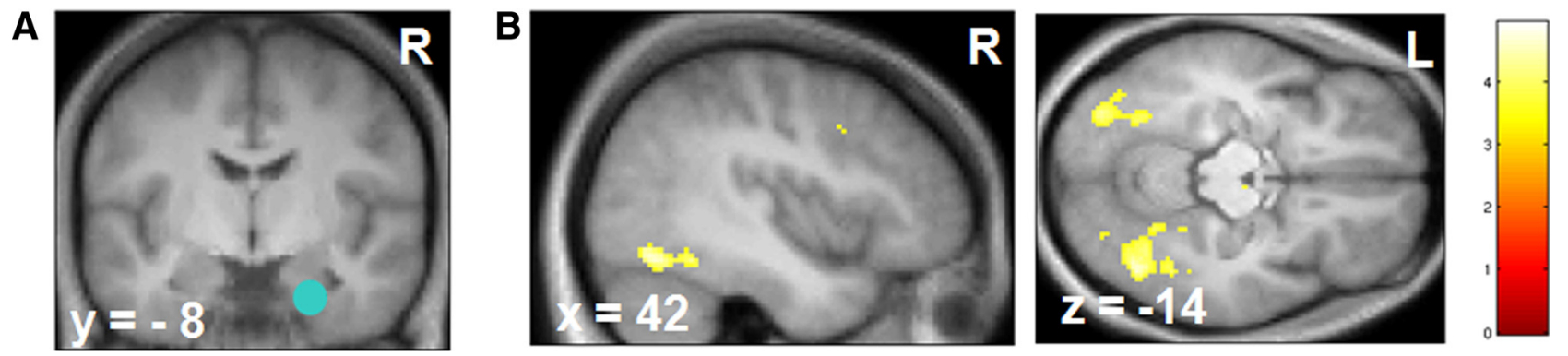

Figure 5. PPI analysis. $A$, The right anterior hippocampus ("source") was defined as a sphere (10 mm diameter) centered around the peak voxel $[(x, y, z)=(24,-8,-30)]$. This particular region showed a pain-specific modulation as revealed by the contrast [(pain + pic) $<$ (tone + pic); see Results]. $B, A$ PPI revealed a reduction in functional connectivity between the right anterior hippocampus and bilateral extrastriate regions (corresponding to the LOC and fusiform gyri) during painful stimulation compared with auditory stimulation. $T$ maps are overlaid on the group mean T1-weighted image. For visualization purposes, thresholded at $p<0.001$ uncorrected. R, Right; L, left.

In everyday life, we are constantly exposed to simultaneous input from different sensory modalities. In this complex environment, the different stimuli commonly compete for limited processing resources (Pashler, 1998). In this competition, visual stimuli are often prioritized, presumably because they provide the most reliable information about the external environment compared with other modalities (e.g., auditory; Molholm et al., 2004; Yuval-Greenberg and Deouell, 2009). This dominance of visual processing is, for instance, reflected in higher recognition rates for visual compared with auditory, haptic, or kinesthetic stimuli during unimodal and bimodal presentation (Busse et al., 2005; Johnson and Zatorre, 2005; Cohen et al., 2009). However, the neutral visual stimuli (which are task relevant but of rather low priority) directly compete with the arousing high-priority nociceptive stimuli. According to the Arousal-Biased Competition Theory (Mather and Sutherland, 2011), the painful stimuli should take precedence over the less arousing visual stimuli and result in impaired perception and memory for the low-priority visual stimuli.

Here we show that the processing of visual stimuli is more sensitive to interference from nociceptive than from auditory stimuli, as indicated in the lower recognition performance during painful stimulation. Importantly, the preference for nociceptive stimuli could not be explained by the unpleasantness, which was matched for nociceptive and auditory stimuli. Although this finding requires additional investigation, we interpret the preferred processing of painful stimuli as the result of the unique link between pain and information about the integrity of our internal environment, which is prioritized over any information from the outside world.

\section{Concurrent pain reduced connectivity between the hippocampus and extrastriate areas}

To explore the mechanisms by which pain specifically interferes with visual encoding, we conducted a PPI analysis to reveal differences in connectivity between the hippocampus and other brain regions depending on the experimental condition. We found reduced functional connectivity of the right anterior hippocampus with extrastriate regions along the ventral stream (extending into the LOC and fusiform gyri) during painful compared with auditory stimulation. Interestingly, reduced connectivity was also observed between the hippocampus and an area in the primary visual cortex (BA17), although to a lesser extent.

This finding is in accordance with the concept that the MTL is highly interconnected with sensory cortices, including ventral visual areas (Lavenex and Amaral, 2000). Different MTL subregions (i.e., perirhinal cortex, lateral entorhinal cortex) receive input from higher visual areas (e.g., fusiform gyrus, LOC; Eichenbaum et al., 2007). During encoding, memory-related MTL regions have been shown to interact with different neocortical areas in a modality- and task-specific manner (Cabeza and Nyberg, 2000). Thus, visual encoding does not only rely on the integrity of the MTL but also on the untainted exchange of information between MTL structures (i.e., hippocampus) and ventral extrastriate regions, in particular the fusiform and lingual gyrus as shown 
in visual identification and categorization tasks (Köhler et al., 1998; Grady et al., 2003). Together, the results of our PPI analysis indicate that the successful interplay of medial temporal and extrastriate regions during visual encoding is more sensitive to the disruptive influence of noxious heat than to unpleasantnessmatched auditory stimuli.

In contrast to our previous study (Bingel et al., 2007), neither pain nor tone stimuli directly modulated activity in object processing areas in the ventral visual stream. We assume that the difference in experimental design could explain these divergent results. The present study used an event-related design, in which the type of aversive stimulation applied in the next trial was unpredictable for the subjects. In contrast, the previous study used a block design in which 10 consecutive trials of the same type were applied. Because predictability critically determines top-down mechanisms such as anticipation and expectancy of the applied stimuli (Porro et al., 2002; Carlsson et al., 2006), the trial-by-trial randomized presentation order of pain and tone stimuli in the present study might have reduced the interruptive effect of pain on primary visual areas. Future studies are required to investigate the interaction between bottom-up and top-down modulatory influences in the interruptive function of pain and other aversive stimuli on MTL and extrastriate regions, for example, depending on the onset predictability of the aversive stimuli.

In summary, our observations extend findings from previous studies by showing that pain not only interferes with regional task-specific activity (i.e., hippocampal activity) but also alters the connectivity between task-related areas, such as extrastriate regions and MTL, suggesting that pain modulates the propagation of information from sensory to memory-related brain regions.

\section{Conclusion}

Taken together, our findings show a pain-specific impairment of visual encoding and subsequent recognition performance. This behavioral effect was augmented in high pain catastrophizers. Our fMRI data indicate that the interruptive effect of pain was mediated by a pain-specific modulation of the right anterior hippocampus as well as a pain-specific reduction in functional connectivity of this region with extrastriate areas (fusiform gyrus, LOC). In sum, these results suggest a pain-specific disruption of visual encoding at the transition from sensory information into memory that occurs over and above the unpleasantness of the stimulus.

\section{References}

Apkarian AV, Bushnell MC, Treede RD, Zubieta JK (2005) Human brain mechanisms of pain perception and regulation in health and disease. Eur J Pain 9:463-484. CrossRef Medline

Bingel U, Rose M, Gläscher J, Büchel C (2007) fMRI reveals how pain modulates visual object processing in the ventral visual stream. Neuron 55: 157-167. CrossRef Medline

Bishop SR, Pivik J, Sullivan MJ (1995) The Pain Catastrophizing Scale: development and validation. Psychol Assess 7:524-532. CrossRef

Brewer JB, Zhao Z, Desmond JE, Glover GH, Gabrieli JD (1998) Making memories: brain activity that predicts how well visual experience will be remembered. Science 281:1185-1187. CrossRef Medline

Busse L, Roberts KC, Crist RE, Weissman DH, Woldorff MG (2005) The spread of attention across modalities and space in a multisensory object. Proc Natl Acad Sci U S A 102:18751-18756. CrossRef Medline

Cabeza R, Nyberg L (2000) Neural bases of learning and memory: functional neuroimaging evidence. Curr Opin Neurol 13:415-421. CrossRef Medline

Carlsson K, Andersson J, Petrovic P, Petersson KM, Ohman A, Ingvar M (2006) Predictability modulates the affective and sensory-discriminative neural processing of pain. Neuroimage 32:1804-1814. CrossRef Medline
Chen AC, Niddam DM, Arendt-Nielsen L (2001) Contact heat evoked potentials as a valid means to study nociceptive pathways in human subjects. Neurosci Lett 316:79-82. CrossRef Medline

Cohen MA, Horowitz TS, Wolfe JM (2009) Auditory recognition memory is inferior to visual recognition memory. Proc Natl Acad Sci U S A 106: 6008-6010. CrossRef Medline

Crombez G, Eccleston C, Baeyens F, van Houdenhove B, van den Broeck A (1999) Attention to chronic pain is dependent upon pain-related fear. J Psychosom Res 47:403-410. CrossRef Medline

Dick BD, Rashiq S (2007) Disruption of attention and working memory traces in individuals with chronic pain. Anesth Anatl 104:1223-1229. CrossRef Medline

Dick BD, Verrier MJ, Harker KT, Rashiq S (2008) Disruption of cognitive function in fibromyalgia syndrome. Pain 139:610-616. CrossRef Medline

Dunsmoor JE, Martin A, LaBar KS (2012) Role of conceptual knowledge in learning and retention of conditioned fear. Biol Psychol 89:300-305. CrossRef Medline

Eccleston C, Crombez G (1999) Pain demands attention: a cognitiveaffective model of the interruptive function of pain. Psychol Bull 125: 356-366. CrossRef Medline

Eichenbaum H, Yonelinas AP, Ranganath C (2007) The medial temporal lobe and recognition memory. Annu Rev Neurosci 30:123-152. CrossRef Medline

Friston KJ, Buechel C, Fink GR, Morris J, Rolls E, Dolan RJ (1997) Psychophysiological and modulatory interactions in neuroimaging. Neuroimage 6:218-229. CrossRef Medline

Friston KJ, Holmes AP, Worsley KJ (1999) How many subjects constitute a study? Neuroimage 10:1-5. CrossRef Medline

Fruhstorfer H, Lindblom U, Schmidt WC (1976) Method for quantitative estimation of thermal thresholds in patients. J Neurol Neurosurg Psychiatry 39:1071-1075. CrossRef Medline

Grace GM, Nielson WR, Hopkins M, Berg MA (1999) Concentration and memory deficits in patients with fibromyalgia syndrome. J Clin Exp Neuropsychol 21:477-487. CrossRef Medline

Grady CL, McIntosh AR, Craik FI (2003) Age-related differences in the functional connectivity of the hippocampus during memory encoding. Hippocampus 13:572-586. CrossRef Medline

Grill-Spector K, Kourtzi Z, Kanwisher N (2001) The lateral occipital complex and its role in object recognition. Vision Res 41:1409-1422. CrossRef Medline

Grisart J, Van der Linden M, Bastin C (2007) The contribution of recollection and familiarity to recognition memory performance in chronic pain patients. Behav Res Ther 45:1077-1084. CrossRef Medline

Grisart JM, Plaghki LH (1999) Impaired selective attention in chronic pain patients. Eur J Pain 3:325-333. CrossRef Medline

Hautzinger M, Bailer M (1993) Allgemeine Depressionsskala. Weinheim: Beltz.

Henson R (2005) A mini-review of fMRI studies of human medial temporal lobe activity associated with recognition memory. Q J Exp Psychol B 58:340-360. Medline

Hirano Y, Fujita M, Watanabe K, Niwa M, Takahashi T, Kanematsu M, Ido Y, Tomida M, Onozuka M (2006) Effect of unpleasant loud noise on hippocampal activities during picture encoding: an fMRI study. Brain Cogn 61:280-285. CrossRef Medline

Jelici M, Geraerts E, Merckelbach H, Guerrieri R (2004) Acute stress enhances memory for emotional words, but impairs memory for neutral words. Int J Neurosci 114:1343-1351. CrossRef Medline

Johnson JA, Zatorre RJ (2005) Attention to simultaneous unrelated auditory and visual events: behavioral and neural correlates. Cereb Cortex 15:1609-1620. CrossRef Medline

Jongsma ML, Postma SA, Souren P, Arns M, Gordon E, Vissers K, WilderSmith O, van Rijn CM, van Goor H (2011) Neurodegenerative properties of chronic pain: cognitive decline in patients with chronic pancreatitis. PloS One 6:e23363. CrossRef Medline

Kim H (2011) Neural activity that predicts subsequent memory and forgetting: a meta-analysis of 74 fMRI studies. Neuroimage 54:2446-2461. CrossRef Medline

Kirschbaum C, Wolf OT, May M, Wippich W, Hellhammer DH (1996) Stress- and treatment-induced elevations of cortisol levels associated with impaired declarative memory in healthy adults. Life Sci 58:1475-1483. CrossRef Medline 
Köhler S, Moscovitch M, Winocur G, Houle S, McIntosh AR (1998) Networks of domain-specific and general regions involved in episodic memory for spatial location and object identity. Neuropsychologia 36:129-142. CrossRef Medline

Kuhajda MC, Thorn BE, Klinger MR, Rubin NJ (2002) The effect of headache pain on attention (encoding) and memory (recognition). Pain 97: 213-221. CrossRef Medline

Lautenbacher S, Huber C, Kunz M, Parthum A, Weber PG, Griessinger N, Sittl R (2009) Hypervigilance as predictor of postoperative acute pain: its predictive potency compared with experimental pain sensitivity, cortisol reactivity, and affective state. Clin J Pain 25:92-100. CrossRef Medline

Laux L, Glanzmann P, Schaffner P, Spielberger CD (1992) Das State-TraitAngstinventar (Testmappe mit Handanweisung, Fragebogen STAI-G Form X1 und Fragebogen STAI-G Form X2). Weinheim: Beltz.

Lavenex P, Amaral DG (2000) Hippocampal-neocortical interaction: a hierarchy of associativity. Hippocampus 10:420-430. CrossRef Medline

Le Pera D, Valeriani M, Niddam D, Chen AC, Arendt-Nielsen L (2002) Contact heat evoked potentials to painful and non-painful stimuli: effect of attention towards stimulus properties. Brain Topogr 15:115-123. CrossRef Medline

Luerding R, Weigand T, Bogdahn U, Schmidt-Wilcke T (2008) Working memory performance is correlated with local brain morphology in the medial frontal and anterior cingulate cortex in fibromyalgia patients: structural correlates of pain-cognition interaction. Brain 131:3222-3231. CrossRef Medline

Mather M, Sutherland MR (2011) Arousal-biased competition in perception and memory. Perspect Psychol Sci 6:114-133. CrossRef Medline

McCracken LM (1997) "Attention" to pain in persons with chronic pain: a behavioral approach. Behav Res Ther 28:271-284.

McCracken LM, Iverson GL (2001) Predicting complaints of impaired cognitive functioning in patients with chronic pain. J Pain Symptom Manage 21:392-396. CrossRef Medline

McCracken LM, Zayfert C, Gross RT (1992) The Pain Anxiety Symptoms Scale: development and validation of a scale to measure fear of pain. Pain 50:67-73. CrossRef Medline

Molholm S, Ritter W, Javitt DC, Foxe JJ (2004) Multisensory visualauditory object recognition in humans: a high-density electrical mapping study. Cereb Cortex 14:452-465. CrossRef Medline

Oosterman JM, Derksen LC, van Wijck AJ, Veldhuijzen DS, Kessels RP (2011) Memory functions in chronic pain: examining contributions of attention and age to test performance. Clin J Pain 27:70-75. CrossRef Medline

Park DC, Glass JM, Minear M, Crofford LJ (2001) Cognitive function in fibromyalgia patients. Arthritis Rheum 44:2125-2133. CrossRef Medline

Pashler HE (1998) The psychology of attention. Cambridge, MA: Massachusetts Institute of Technology.

Patil PG, Apfelbaum JL, Zacny JP (1995) Effects of a cold-water stressor on psychomotor and cognitive functioning in humans. Physiol Behav 58: 1281-1286. CrossRef Medline

Peters ML, Vlaeyen JW, Kunnen AM (2002) Is pain-related fear a predictor of somatosensory hypervigilance in chronic low back pain patients? Behav Res Ther 40:85-103. CrossRef Medline

Porro CA, Baraldi P, Pagnoni G, Serafini M, Facchin P, Maieron M, Nichelli P (2002) Does anticipation of pain affect cortical nociceptive systems? J Neurosci 22:3206-3214. Medline
Powell HW, Koepp MJ, Symms MR, Boulby PA, Salek-Haddadi A, Thompson PJ, Duncan JS, Richardson MP (2005) Material-specific lateralization of memory encoding in the medial temporal lobe: blocked versus event-related design. Neuroimage 27:231-239. CrossRef Medline

Radloff LS (1977) The CES-D Scale. Appl Psychol Measure 1:385-401. CrossRef

Rainer G, Augath M, Trinath T, Logothetis NK (2001) Nonmonotonic noise tuning of BOLD fMRI signal to natural images in the visual cortex of the anesthetized monkey. Curr Biol 11:846-854. CrossRef Medline

Rolke R, Baron R, Maier C, Tölle TR, Treede RD, Beyer A, Binder A, Birbaumer N, Birklein F, Bötefür IC, Braune S, Flor H, Huge V, Klug R, Landwehrmeyer GB, Magerl W, Maihöfner C, Rolko C, Schaub C, Scherens A, Sprenger T, Valet M, Wasserka B (2006) Quantitative sensory testing in the German Research Network on Neuropathic Pain (DFNS): standardized protocol and reference values. Pain 123:231-243. CrossRef Medline

Rose M, Schmid C, Winzen A, Sommer T, Büchel C (2005) The functional and temporal characteristics of top-down modulation in visual selection. Cereb Cortex 15:1290-1298. CrossRef Medline

Schnurr RF, MacDonald MR (1995) Memory complaints in chronic pain. Clin J Pain 11:103-111. CrossRef Medline

Schwarze U, Bingel U, Sommer T (2012) Event-related nociceptive arousal enhances memory consolidation for neutral scenes. J Neurosci 32:14811487. CrossRef Medline

Seminowicz DA, Davis KD (2007a) Pain enhances functional connectivity of a brain network evoked by performance of a cognitive task. J Neurophysiol 97:3651-3659. CrossRef Medline

Seminowicz DA, Davis KD (2007b) Interactions of pain intensity and cognitive load: the brain stays on task. Cereb Cortex 17:1412-1422. CrossRef Medline

Spielberger CD, Gorssuch RL, Lushene PR, Vagg PR, Jacobs GA (1983) Manual for the state-trait anxiety inventory. Palo Alto, CA: Consulting Psychologists.

Tiemann L, Schulz E, Gross J, Ploner M (2010) Gamma oscillations as a neuronal correlate of the attentional effects of pain. Pain 150:302-308. CrossRef Medline

Van Damme S, Crombez G, Eccleston C (2004) Disengagement from pain: the role of catastrophic thinking about pain. Pain 107:70-76. CrossRef Medline

Vancleef LM, Peters ML (2006a) The interruptive effect of pain on attention. J Pain 7:21-22. CrossRef Medline

Vancleef LM, Peters ML (2006b) Pain catastrophizing, but not injury/illness sensitivity or anxiety sensitivity, enhances attentional interference by pain. J Pain 7:23-30. CrossRef Medline

Walter B, Hampe D, Wild J, Vaitl D (2002) Die Erfassung der Angst vor Schmerzen: Eine modifizierte deutsche Version der Pain Anxiety Symptoms Scale (PASS-D). Der Schmerz 16:83

Weissman-Fogel I, Moayedi M, Tenenbaum HC, Goldberg MB, Freeman BV, Davis KD (2011) Abnormal cortical activity in patients with temporomandibular disorder evoked by cognitive and emotional tasks. Pain 152: 384-396. CrossRef Medline

Yuval-Greenberg S, Deouell LY (2009) The dog's meow: asymmetrical interaction in cross-modal object recognition. Exp Brain Res 193:603-614. CrossRef Medline

Zimmermann P, Fimm B (1992) Testbatterie zur Aufmerksamkeitsprüfung (TAP). Würselen: Psytest. 